This PDF is a simplified version of the original article published in Internet Archaeology. Enlarged images which support this publication can be found in the original version online. All links also go to the online version.

\title{
Digital Dutch Archaeology: Future perspectives
}

Hella Hollander

Digital archaeology in the Netherlands is connected with investments in a new large-scale and innovative Dutch research infrastructure for the next 10 years. Priorities are set to combine forces at a national level in order to contribute to the international position of the Netherlands as a 'knowledge country'. Researchers need to coordinate and collaborate even more to set up a FAIR enabling data infrastructure with limited resources. Regarding the archaeological discipline, the use of formal quality standards and legislation that certifies archaeological organisations to carry out archaeological work improves national collaboration and stimulates the digital workflow. DANS is the dedicated national repository for archaeology in the Netherlands and is launching the Data Station Archaeology, a repository meeting the latest technological standards. Finding and sharing data of high quality facilitates knowledge of archaeological discoveries; a flourishing open access trend in Dutch archaeology stimulates a strong growth in the use of data. To keep up with innovative developments, a growing community of archaeologists and other specialists are working together in international projects to secure the future of European Archaeology.

\section{FAIR Archaeological Data}

This article gives an overview of the current situation regarding digital archaeology in the Netherlands and how it is connected with worldwide innovations. The network of people working according to digital archaeological standards and best practices is growing rapidly: digital working is no longer seen as a starting point, but as a formal standard. There is a need to combine forces at a national level in order to serve the Dutch archaeological community with FAIR data and a FAIR-enabling data infrastructure. In short, this means creating data of high quality, making it easy to find and access and offering it in such a way that it can easily be reused, processed and combined with other (types of) data in a sustainable way. A growing awareness of the need of FAIR data to create a successful Dutch data landscape is visible through 
the whole of Dutch society, from politics to end-users, from data providers to data collectors.

The aim of the Dutch government is to create a clear and efficient national Dutch infrastructure for research data based on local and thematic Digital Competence Centres (DCCs). These DCCs will deploy a services package to help researchers process and deposit their data according to FAIR principles. Archaeology will be part of the DCC for the Humanities and Social Sciences and, being a mature discipline in digital research data management, a best practice example for related disciplines who are organising their research environment according to more formal and national policies. An example of national combined services is the creation of a signpost tool that helps Dutch institutions with formulating their policy on file formats. Via an interactive step-by-step plan, file formats can be investigated. This tool is formed within the Dutch Digital Heritage Network (NDE) by a group of experts in the Netherlands. With their years of experience with preferred formats (and for many formats knowledge based on archaeological practices) the input of DANS, the Dutch National Centre of Expertise and Repository for Research Data, was significantly valued. Paramount is the development of an infrastructure that offers users a seamless experience in their own research environment, while in the background different systems and platforms are being used. Relying on standardised procedures, policies, protocols and best practices is therefore very important.

\section{Formalising National Standards}

Data Archiving and Networked Services (DANS) is the Dutch national expertise centre and repository for research data. The institute covers all scientific disciplines, with a strong foundation in the social sciences and humanities. DANS is an institute of the Royal Netherlands Academy of Arts and Sciences (KNAW), together with the research funding organisation NWO (Dutch Research Council). With the establishment of DANS in 2005, previous initiatives merged together: the Steinmetz archive (dating back to 1964), the Dutch Historical Data Archive (NHDA) and the Scientific Statistical Agency (WSA). In 2021, with more than 180,000 datasets and 60 employees, DANS is one of the leading repositories in Europe. This year, DANS successfully renewed its CoreTrustSeal Data repository Certification. With this internationally recognised quality mark for trusted digital repositories, DANS shows that reliability and sustainability of data are guaranteed. DANS supports researchers, data professionals, other data archives, research institutions and funding bodies with training and advice in the field of data management, certification, FAIR data and open access.

The e-Depot for Dutch archaeology is a collaboration between DANS and the Cultural Heritage Agency of the Netherlands (RCE) and is housed at the DANS national data archive, enabling the digital research data of Dutch archaeologists to remain accessible and usable in the long term. Archaeologists can approach the eDepot to deposit data, or to view and reuse data. Depositing documentation of archaeological research is not voluntary, but is prescribed in legally required protocols: the organisation delivers project documentation (including standard reports and reports of specialists) to the depot holder/owner no later than two years after completion of the fieldwork. The digital project documentation is delivered to the 
depot holder/owner or an e-depot designated by it or - if the depot holder/owner does not have or does not designate an e-depot - to an e-depot to be determined by the organisation (BRL SIKB 4000, versie 4.1). https://www.sikb.nl/archeologie

Formal standards improve this national collaboration. Together with the Environment and Planning Act, The Heritage Act covers museum objects, museums, monuments and archaeology on land and under water. The most important change for archaeologists with the arrival of the Heritage Act in 2016 was the replacement of the excavation permit by a new system based on certification. Archaeologists are obliged to carry out excavations and field surveys according to professional quality standards (KNA) and they may only excavate if their organisation or company is certified. They are expected to carry out archaeological work as described in official assessment guidelines (BRL) and associated protocols. The BRL and legally required protocols are designated by the Minister of Education, Culture and Science (OCW) as mandatory documents. The BRL and KNA require that information must be permanently stored in an e-depot. An e-depot is defined as a certified storage place for durable storage of digital data. Since 2007, DANS offers the community a designated national archaeological archive as the e-Depot for Dutch archaeology that meets the formal requirements.

In 2013, archaeological companies carried out the first experiments to implement a proposed standard in order to be able to digitally exchange data describing artefacts between all archaeological organisations in the Netherlands. The first requirements of this standard were formulated to simplify the delivery and acceptance of archaeological material and documentation. In the years that followed, the standard improved and formalised and became part of the official BRL and the Dutch Quality Control System for Archaeology (KNA). Dutch archaeologists are requested to exchange their data by exporting their project metadata and fieldwork database tables to this national metadata standard (XML) called SIKB0102 (Gilissen and Hollander in prep). To centralise this flow of data, the provincial depots developed ArcheoDepot, a system in which archaeological objects are linked with describing metadata and connected documentation such as text, spreadsheets and images. To guarantee long-term reusability of datasets submitted to the ArcheoDepot, they are therefore automatically added by the provincial depots to the archaeological archive collection of DANS via a machine-to-machine connection.

However, a large amount of the archaeological data was is manually submitted at DANS and not via a machine-to-machine protocol. In such cases, errors occur more often. A data processor will check the quality of the metadata, the readability of the files and the durability of the file format. The original (native) format together with the preservation format (for example csv, mif/mid) are both archived. On the basis of new proposed BRL protocols, submitted digital files must comply with the preferred formats of DANS. DANS has defined a preferred format for each type of file. These are file formats that are internationally trusted to offer the best guarantees for usability, accessibility and sustainability for new or additional research in the long term. When the public round of consultation of this updated BRL ends, this preferred format policy will become part of the formal procedure for archaeologists depositing data. 


\section{Flourishing Open Access Trend}

The Dutch Cultural Heritage Agency (RCE), DANS, depot holders of provinces and municipalities, the commercial archaeological sector, universities and independent archaeologists, all want to be able to share data for which sustainability and reliability is guaranteed. People interested in discoveries that have been made in their neighbourhood, or in a certain thematic topic, are searching through documents and pictures for their private research. The open access trend in Dutch archaeology has become mainstream in recent years. Eight years ago, it started with a few large archaeological companies who opted for more flexible access rights. Instead of only granting access to professional archaeologists or granting access after receiving a permission request for access, these companies opted for access for any user having a registered DANS account. This positive trend continued after the introduction of the Creative Commons licences into the archive, which improved the dissemination and use of licensed materials. At present, $99 \%$ of the archaeological datasets archived at DANS in the trusted e-depot for Dutch archaeology are now directly accessible. The milestone of 100,000 archaeological datasets was reached in 2021 and, of these, 99,000 reports, surveys or excavation archives are directly and publicly accessible. The possibility of sharing high quality data facilitates the generation and answering of completely new research questions. This flourishing open access trend will continue through the growing involvement of the general public in research projects. This will further increase the reuse of data, as this information reservoir will no longer be restricted to a group of researchers but will be used by many people. Provenance of the data source will become more important than ever.

\section{Figures for Reuse}

Tracing the way data is reused by looking into academic literature is difficult, as data citation with references to persistent identifiers of used datasets is not yet common practice. The research of Borgman et al. (2019) showed that data consumers are a diverse group: students, researchers, museum curators, employees of private companies, government employees. The aim of locating and downloading data varies according to their needs. However, more precise figures of reuse of digital data are available from the management tool of the archive of DANS and usage growth is visible. In 2007, the first year when the archiving system of DANS went operational, 750 datasets were downloaded for reuse and this number grew to reach 30,000 in 2013, and increased to almost 40,000 datasets in 2019 (Doorn 2020). Furthermore, a study about the reuse of archaeological data in the Netherlands was undertaken to gain an indication of the implementation of article 7 of the Malta Convention: collection and dissemination of scientific information for the purpose of facilitating the study of, and dissemination of knowledge about, archaeological discoveries (de Bruijn 2019). The download statistics of datasets and files stored in the e-depot for Dutch archaeology at DANS showed a remarkably high percentage of reuse of the data by professionals: of the 74,000 downloaded archaeological datasets in 2017 and 2018, over 52,000 datasets (70\%) were downloaded by archaeological professionals. In those two years in total 253,000 files were downloaded, with an average of around 3.5 files downloaded per dataset. 


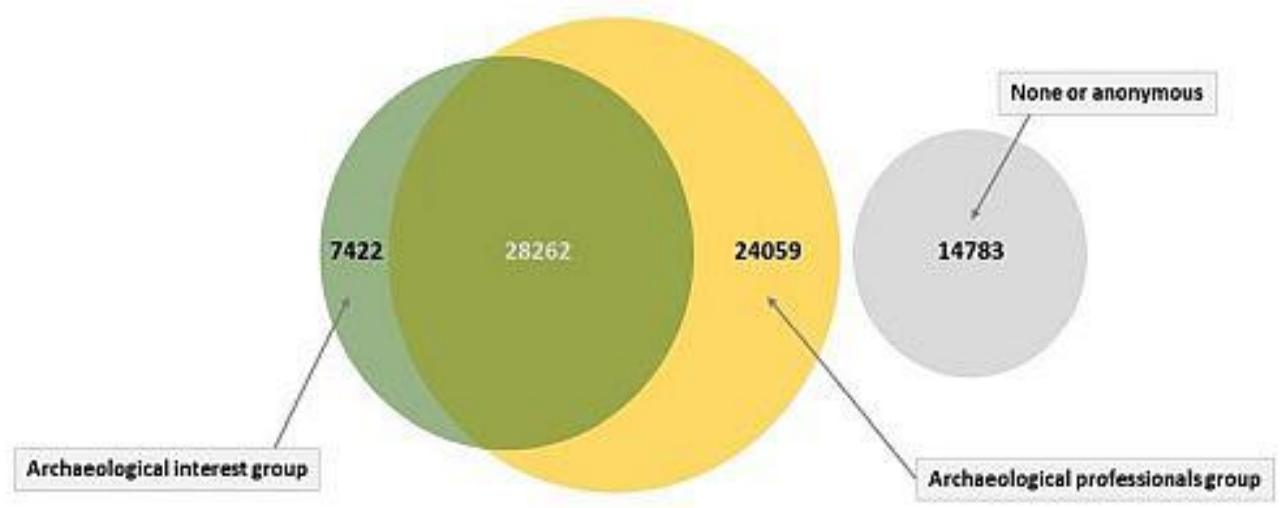

Figure 1: Number of downloads of archaeological datasets divided by account groups

\section{Fast Forward}

In the coming months, DANS is launching a new infrastructure based on domainspecific Data Stations. To guarantee long-term and secure storage of the archaeological collection according to the newest standards, the existing archaeological data archive is being migrated to the Data Station Archaeology. The technology used for this archiving system is based on the open-source DataVerse repository software of Harvard University and has the advantage that developments are done in collaboration with a worldwide, large and growing community. In this renewed national e-depot dedicated to Dutch archaeology, archaeologists can store, share and publish their data collections online, both during their research and after new discoveries. It is a self-depositing system; for support researchers can directly approach the Data Station Manager for archaeology, who is leading this national centre of expertise and manages the repository. A data-processing team share their expertise and offer guidance when needed. Data Station Archaeology is focused on the Dutch research discipline of archaeology but, as a national domain portal, is strongly connected with European portals such as the archaeological ARIADNEplus portal and research infrastructures like E-RIHS. Sharing data online across borders is facilitated. Linking national with international thesauri improves the findability of data and their interoperability. Archaeological data collections from the Netherlands are linked to collections of other archaeological archives in Europe.

The metadata to describe archaeological research in the Data Station Archaeology is associated with discipline-specific terms and thesauri, which improves finding specific information. Analytical tools and applications like visual maps and text mining are part of this ecosystem, improving the reuse of data. By offering research data management training and infrastructural expertise, DANS contributes to several archaeological European projects. Partners within national and international projects exchange best practices and work together on new developments. This knowledge exchange enables DANS to innovate continuously and follow technical developments closely. Exploring methods, standards and practices of depositing and 
retrieving 3D data are investigated in the $4 \mathrm{CH}$ and Pure3D projects. This focus on 3D technology offers DANS the opportunity to contribute to the design and development of an infrastructure that connects to new emerging technologies for the publication and preservation of 3D scholarship. How to archive 3D information in the most sustainable way, how to promote reuse and how to make this special type of data FAIR are questions to be answered. DANS has a leading role in policy making, such as creating an archaeological data management plan (DMP) and promoting the quality of the data. The ARIADNE data management plan (DMP) is based on the $\mathrm{H} 2020$ research data management (RDM) requirements and is integrated and aligned with the protocol for archaeological data management based on the Science Europe core requirements for RDM. An updated DMP template will be compliant with the new Horizon Europe requirements.

DANS is visible as a national expert in services on FAIR and certified data as a member of the Steering Committee of E-RIHS NL, the European Research Infrastructure for Heritage Science (E-RIHS). DANS advises how to organise the data structure and provides a trusted repository for the data collected in the Constructing the Limes project. This project is part of the Dutch Research Agenda and studies the border of the Roman Empire that ran right through the Netherlands. By participating in activities that take place in a broad network of archaeological partners, local archives, museums and cultural heritage organisations, DANS maintains a key position in promoting and offering services and knowledge on research data management. The COST action SEADDA is demonstrating the benefits of a growing international community of archaeologists and digital specialists working together to secure the future of archaeological data across Europe and beyond. The progress is moving forward fast.

\section{Bibliography}

Borgman, Ch.L., Scharnhorst, A. and Golshan, M.S. 2019 'Digital data archives as knowledge infrastructures: mediating data sharing and reuse', Journal of the Association for Information Science and Technology (ASIS\&T) 70(8), 888904. https://doi.org/10.1002/ asi.24172

de Bruijn, C. 2019 Digging Data: How effective is the implementation of article 7 of the Malta convention in the Netherlands currently and does it stimulate the reuse of digital archaeological data in further research?. https://hdl.handle.net/1887/79773

Doorn, P.K. 2020 'Archiving and managing research data: data services to the domains of the humanities and social sciences and beyond: DANS in the Netherlands', Der Archivar 73(1), 44-

50. https://hdl.handle.net/20.500.11755/791a4624-2bfe-4300-b365-6293997b08fo

Gilissen, V. and Hollander, H.S., in prep. 'My data manager is a robot!' 
